\title{
Applying the Flow-interception Location Model to Select the Location of Construction Industrial Yard
}

\author{
Jinxi JING ${ }^{1 *}$, Kaijian $\mathrm{LI}^{2}$, Guiwen $\mathrm{LIU}^{3}$, Pengpeng XU ${ }^{4}$ \\ ${ }^{1}$ Master Degree Candidate, Faculty of Construction Management and Real Estate, Chongqing \\ University \\ ${ }^{2}$ PhD candidate, Faculty of Construction Management and Real Estate, Chongqing University \\ ${ }^{3}$ Professor, Faculty of Construction Management and Real Estate, Chongqing University \\ ${ }^{4}$ Associate Professor, Faculty of Construction Management and Real Estate, Chongqing \\ University \\ *Corresponding author's e-mail: jinxi.jing@yahoo.com
}

\begin{abstract}
Construction industrialization is a remarkable innovation and improvement in construction industry. This new construction method can address the drawbacks of conventional one, which has extensive mode of production, lower labour productivity and serious energy consumption and environmental pollution. Recent years, the construction industrial yard emerges as a method to promote the modernization of construction industry in China. However, there has been little to no empirical literature discussed the method to identify the location of construction industrial yard. The location problem is one of key factors to affect whether the yard can be sustainable in economic, social and environmental profits. In order to make the location of construction industrial yard more scientific and meet the special requirements of construction industry, this paper proposes a bi-level model based on the flow-interception location model (FILM). In the location model, the transport network is divided into sub-networks of raw materials and products respectively. The upper-level model is assumed to make the decision about the location and the origin-destination (OD) matrices of raw materials and products flow. The lower-level is used to calculate the flow of each section under given OD flow matrices, and gives feedback to the upper model. An efficient heuristic method is introduced to solve this bi-level model. This bi-level model of construction industrial yard can help planners make a more scientific decision when establishing a new yard and promote the development of construction industrialization.
\end{abstract}

\section{KEYWORDS}

Construction industrialization; Location; Construction industrial yard; Flow interception location model

\section{INTRODUCTION}

It is considered that construction industrialization is an inevitable trend in China. The experience in the achieved construction industrialization counties indicates that construction industrialization can save construction time, improve building quality, increase labour 
productivity, and save energy (Ning \& Yingbo, 2010).In order to promote construction industrialization, Ministry of Housing Urban-Rural Development (MOHURD) approved the establishment of "National Housing Industrialization Base" in 2001. And five years later, the MOHURD issued National Housing Industrialization Base Pilot Scheme, which indicated the formal implementation of National Housing Industrialization Base. The MOHURD are currently engaged in promoting national pilot programs. Up to now 59 bases have been established, and 11 cities have approved to be "National Housing Modernization Pilot City" in China (MOHURD, 2015).

For a construction industrialization yard, the location is the key to its success since it can affect whether the yard can be sustainable in economic, social and environmental profits. And construction logistics cost is the key factor affecting the location decision of construction industrialization yard. Previous studies have discussed the potential barriers for implementing construction. For instance, Rahman (2014) argued that the logistics problem was the major technical barrier (e.g., the limitation in transporting large prefabricated components). Fang and $\mathrm{Ng}$ (2011) pointed out that without careful planning and controlling on the flow of construction materials, it is possible that the cost of materials may increase unnecessarily. Jaillon and Poon (2008) identified that higher initial costs along with the transportation costs of prefabricated components to be the two major economic constraints. Thus, the logistics is the main point to select the location of construction industrial yard, and the flow of raw materials and products also should be well planned. By attaining the research objectives, this study proposed a bi-level model of construction industrial yard based on the flow-interception location model, which can help planners make a more scientific decision when establishing a new yard.

\section{THE CONSTRUCTION INDUSTRIAL YARD}

A construction industrial yard is the combination of an industrialization base and eco-industrial yard. And it represents a practical way to apply the concept of industrial symbiosis. Construction industrial yard has gained popularity in promoting construction industrialization in China, and China has achieved encouraging progress.

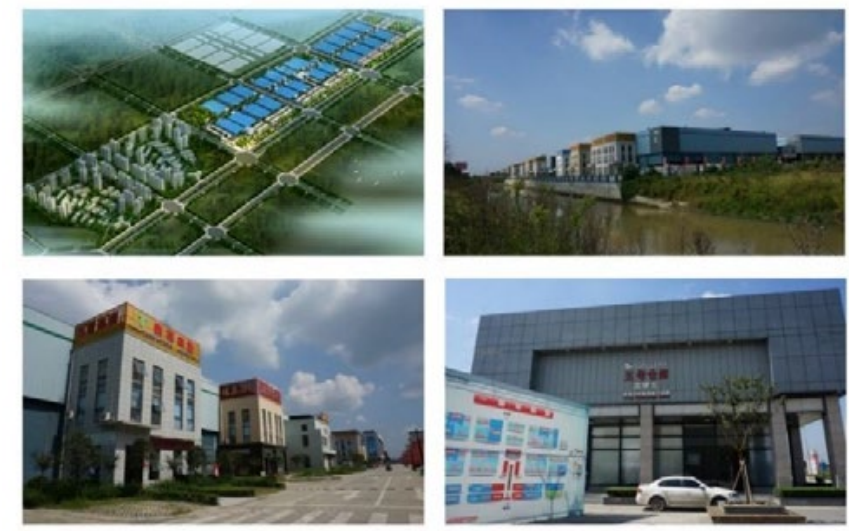

Figure 1. Chengdu Jiangong Construction Yard (source: http://www.yardchain.com/home.asp). 


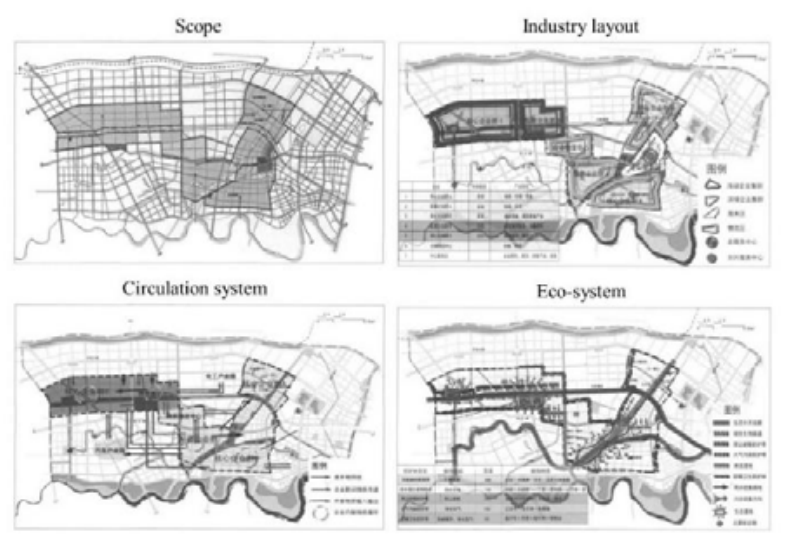

Figure 2. Shenyang Modern Construction Yard [adapted from(li, Zhu, Zhang, \& Hu, 2011)].

The construction industrial yard is supported by the government in China. Through the preferential policies, construction companies that are engaged in construction structures and fixtures, construction equipment, construction materials or site construction, and technology companies that are specialized in industrial research and design or construction technology, and service-oriented companies that provide construction logistics and warehousing, are attracted to settle into the yard. The yard forms a complete industrial chain and plays a good leading and exemplary role in the local and national level to promote the construction industry.

\section{METHODOLOGY}

\section{The classifications of location models}

Location problems are often created by the lack of spatial resources which creates the allocation problems. In order to be successful, management must investigate where to physically locate a set of facilities (resources) (khaled \& Kim, 2012). Eiselt (2008) mentioned seven types of location models. These are: minisum problems, minimax problems, undesirable facilities models, probabilistic location models, hierarchical location models, competitive location models, location-routing problems and location models with equity objectives. Since the network become more and more complicated, many new facility location models have been put forward, such as the flow interception location model, which tries to determine the location and allocation of the resource points in order to serve some specified demand points.

\section{The flow interception location model}

Hodgson (1990) and Berman, Larson, and Fouska (1992)presents the flow interception problem (FIP) and developed a heuristic greedy algorithm to solve the FIP. The basic problem of FIP is to locate $m$ facility to intercept as much flow as possible from a given set of pre-existing flows on the network. It assumes the 'interception' occurs if a flow passes through at least one facility.

FILM is a fruitful garden for research. Since the early 1990s, over 30 different FILMs have appeared. In these publications, location researchers have developed new models by introducing changes in the objectives functions, constraints, and/or assumptions. These changes have led to many disparate models, each requiring a somewhat different solution method, and they have challenged the development of standardized software that would encourage widespread use in real-world, strategic decision-making processes (Zeng, Castillo, \& Hodgson, 2010). In the study 
of Wang (2008), two new models based the flow intercepting spatial interaction model are formulated to locate competitive service facilities under the scenario-based market demand uncertainty. Hu, Yang, and Yang (2009) studied location problems with congestion demand and a bi-objective model based on flow interception problem was proposed. Wu, Yang, and Dong (2014) optimized the location problem of the manufacturing industry in the context of the economic globalization, and formulates the bi-level programming model combining both the flow-interception location and the traffic assignment.

There is a big difference of location problem between traditional facility and the construction industrial yard. The network of yard involves in the transport of two substances, namely the transportation of raw materials from the supplies to the yard and the transportation of products from the yard to the construction site. The construction logistics cost and the flow of raw materials and products are the key factors affecting the location decision of construction industrial yard. Thus, more attention should be paid on the yard location in a network where demand is exerted by traffic flowing between origins (raw materials suppliers) and destinations (construction sites).

The Construction Industrial Yard can been seen as a facility which provides services in the network. The manufacturing process of raw materials into products, for example prefabricated building components, in construction industrial yard can be abstracted by the process of providing the service to the substance which flow through the path from raw materials suppliers to construction site, and the raw materials and products are the initial and the final form of this substance.

\section{LOCATION MODEL OF CONSTRUCTION INDUSTRIAL YARD}

\section{The basic idea of location model of construction industrial yard}

The manufacturing process of the yard involves three links that are (i) raw material procurement,(ii) precast components built as types of pieces, units, or modules in the yard (e.g., floor slab, facades, staircases, beams, bathrooms, kitchens and so on), (iii) transportation of prefabricated components to construction sites.

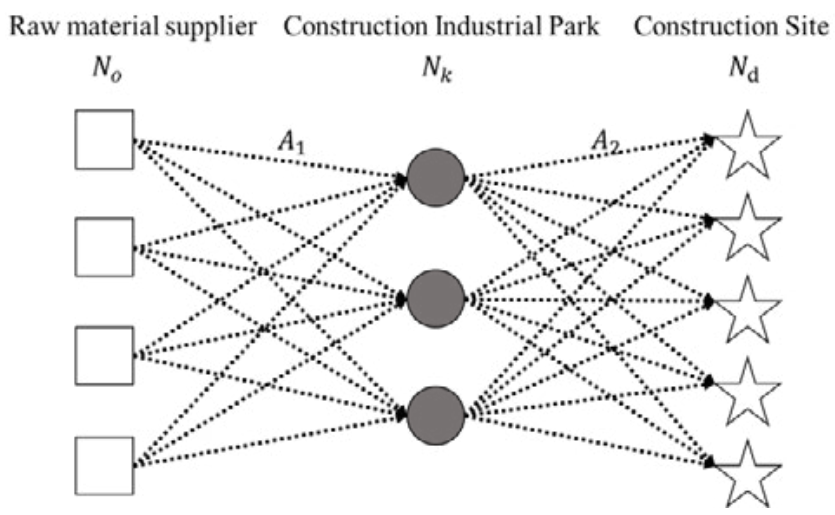

Figure 2. Simplified transport network

The focus of original FILM is on maximizing the total consumption of the service. And in this study, the attention is paid to the access to low-cost and path optimization. So the location model of construction industrial yard is a bi-level programming problem that involves the FIP and the 
allocation of flow under given OD matrices. In the model, the transport network is divided into sub-networks of raw materials and construction components respectively (see in figure 2).

Where the parameters are: $N_{o}$ is the set of raw material suppliers; $N_{d}$ is the set of construction sites; $N_{k}$ is the set of potential location of construction industrial yard; $A_{1}$ is the set of sections in the raw material network; $A_{2}$ is the set of sections in the product network; $\psi_{i k}$ is the set of paths from suppier $i$ to yard $k ; \varphi_{k j}$ is the set of path from yard $k$ to construction $j$.

\section{The upper-level location model}

The upper-level is assumed to make the decisions about the location in order to maximize the total flow served by yard $\mathrm{k}$ and minimize the transportation cost. The upper-level for the yard location problem can be expressed as follows:

$$
\operatorname{Min} F_{0}=\sum_{a} x_{a} \cdot C_{a}\left(x_{a}\right)+\sum_{b} x_{b} \cdot C_{b}\left(x_{b}\right)
$$

s.t.

$$
\begin{aligned}
& q_{i k}=O_{i} \cdot \frac{y_{k} \cdot \exp \left(-\mu \cdot u_{i k}\right)}{\sum_{l} y_{l} \cdot \exp \left(-\mu \cdot u_{i l}\right)}, \forall i \in N_{0}, k \in N_{l} \\
& \eta \cdot \sum_{i} q_{i k}=G_{K}, \forall k \in N_{l} \\
& Q_{k j}=A_{k} \cdot B_{j} \cdot G_{k} \cdot D_{j} \cdot \exp \left(-\xi \cdot v_{k j}\right), \forall k \in N_{l} j \in N_{d} \\
& A_{k}=\frac{1}{\sum_{j} B_{j} \cdot D_{j} \cdot \exp \left(-\xi \cdot v_{k j}\right)}, \forall k \in N_{l} \\
& B_{j}=\frac{1}{\sum_{k} A_{k} \cdot G_{k} \cdot \exp \left(-\xi \cdot v_{k j}\right)}, \forall j \in N_{l} \\
& y_{k} \in\{0,1\}, \forall k \in N_{l}
\end{aligned}
$$

Where $\mathrm{y}_{k}=1$, if the yard is located at $k$ or 0 , otherwise; $q_{i k}$ is material flow of OD pair in network of raw materials; $Q_{i k}$ is product flow of OD pair in network of products; $O_{i}$ is the production of raw material supplier $i$; $D_{j}$ is the demand of construction site $j ; x_{a}$ is the flow of section $a$ in network of raw materials; $f_{i k m}$ is the flow of $m^{\text {th }}$ path from supplier $i$ to yard $k$ in network of raw materials; $\delta_{a, m}^{i k}=1$, if section $a$ is on the $m^{\text {th }}$ path in network of raw materials; 0 , otherwise. $C_{a}(\cdot)$ is the congestion function of section $a$ in network of raw materials (Gürel, 2011); $x_{b}$ is the flow of section $b$ in network of product; $g_{k j n}$ is the flow of $n^{\text {th }}$ path from yard $k$ to construction site $j$ in network of product; $\delta_{b, n}^{k j}=1$, if section $b$ is on the $n^{\text {th }}$ path in network of product; 0 , otherwise. $C_{b}(\cdot)$ is the congestion function of section $b$ in network of product (Gürel, 2011); $u_{i k}$ is the lowest transportation cost from supplier $i$ to yard $k$ in network of raw materials; $v_{k j}$ is the lowest transportation cost from yard $k$ to construction site $j$ in network of product.

The objective function (1) is aimed at lowering transportation cost as much as possible. Constraint (2) (4) is used to calculate the OD matrices of raw materials based on the gravity model. Constraint (3) indicates that the production scale of yard $k$ is determined by the sum of raw materials flow. Constraint (7) enforces the $\mathrm{y}_{k}$ variables to be $0-1$. 


\section{The lower-level allocation model}

It is worth emphasizing that the FIP problem must be solved with the network flow pattern. In general, the location strategy will definitely induce changes in flow over the network. Traditionally, the basic FIP models hypothesize that the flow on each path is given and fixed. In this bi-level programming model, the flow of each OD pair is assumed to follow model under the given location project. The lower-level model for the flow allocation of raw materials network can be expressed as follows:

$$
\operatorname{Min} F_{1}=\sum_{a} \int_{0}^{x_{a}} C_{a}(\omega) d \omega
$$

s.t

$$
\begin{aligned}
& \sum_{m} f_{i k m}=q_{i k}, \forall i \in N_{o}, k \in N_{k} \\
& f_{i k m} \geq 0, \forall i \in N_{o}, k \in N_{k}, m \in \psi_{i k} \\
& x_{a}=\sum_{i} \sum_{k} \sum_{m} f_{i k m} \delta_{a, m}^{i k}, \forall a \in A_{1}
\end{aligned}
$$

The lower-level model for the flow allocation of product network can be expressed as follows:

$$
\operatorname{Min} F_{2}=\sum_{b} \int_{0}^{x_{b}} C_{b}(\omega) d \omega
$$

s.t.

$$
\begin{aligned}
& \sum_{n} g_{k j n}=Q_{i k}, \forall k \in N_{k}, j \in N_{d} \\
& g_{k j n} \geq 0, \forall k \in N_{k}, j \in N_{d}, n \in \psi_{k j} \\
& x_{b}=\sum_{k} \sum_{j} \sum_{n} g_{k j n} \delta_{b, n}^{k j}, \forall b \in A_{2}
\end{aligned}
$$

The objective function (8) is aimed at making a balanced distribution of flow. Constraint (9) indicates the total flow of any OD pair in the raw materials network is the sum of all paths flow. Constraint (11) states the total flow of section $a$ is the sum flow of all paths. The objective function and constraints of lower-level model for the flow allocation of product network is the same as the raw material network.

The interactive feedback relationship between the upper and lower model are as follows: firstly, through optimization the upper model gives the location plan and the OD matrices of raw materials and products, which are input to the lower model; then the lower model distributes the flow of each section and calculate its congestion, and gives feedback to the upper model; then the upper model revises its objective function value based on the output of the lower model before recalculating. This process will be iterative until equilibrium is reached

\section{The heuristic algorithm based on genetic and Frank-Wolfe algorithm}

As we all known, a bi-level programming model is an NP-hard problem. To solve this model, this study applies the heuristic algorithm based genetic and Frank-Wolfe algorithm which is designed by (Wu, Yang, \& Dong, 2015). The algorithm process is shown in Fig. 3 


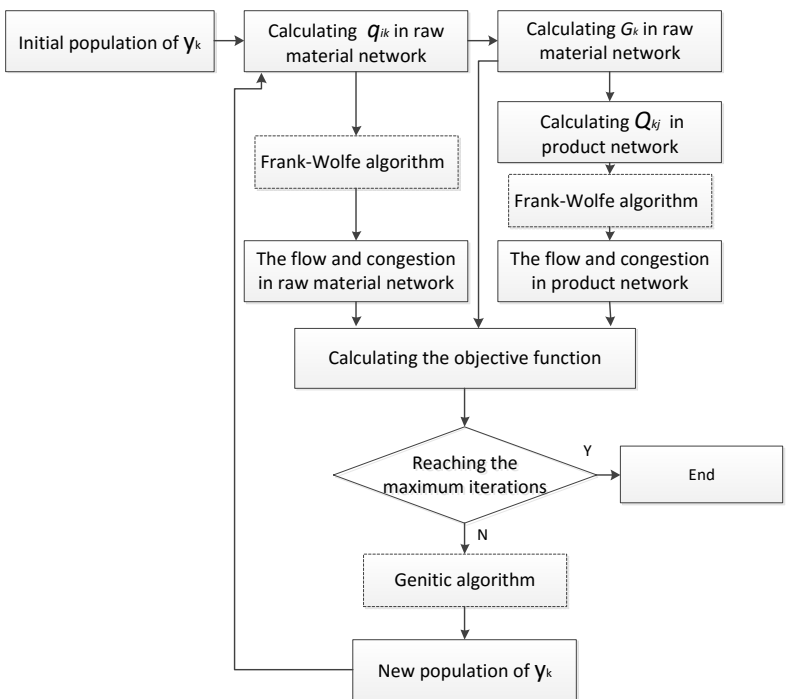

Figure 3. Process flowchart

\section{A small demo case}

Consider the small-sized example network with seven nodes shown in Fig. 4.

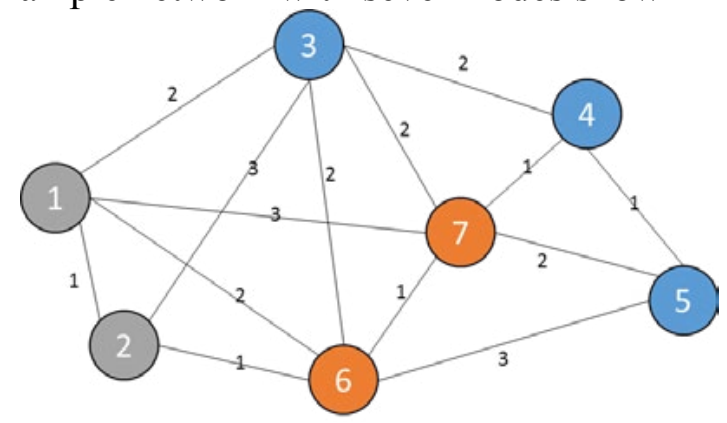

Figure 4. Example network

In this network, node 1, 2 represent raw material suppliers; node 3, 4, 5 represent construction sites; node 6, 7 represent potential location of construction industrial yards. Length information for each path is given in figure 4 . Suppose that $O_{1}=12, O_{2}=18, D_{1}=2, D_{2}=3, D_{3}=5, \mathrm{C}(\mathrm{x})=7+$ $0.04 \mathrm{x}, \lambda=0.05, \eta=0.7, \mu=0.05, \xi=0.05$. The proposed mathematical model, The heuristic algorithm were applied to this example network. The solution quality of the greedy algorithm is $94.77 \%$ of the mathematical model and the result indicates that node 6 is more suitable to establish a construction industrial yard.

\section{CONCLUSION}

This paper has attempted to address the location problem of the construction industrial yard. A bi-level location model based the flow interception model are formulated in this study to tackle the issue of locating the construction industrial yard. Traditional FILM are used to select service locations such that the flows that are intercepted are maximized from a given set of pre-existing flows on the network. In this study, the bi-level location model avoid the problem that the flows and paths should be determined in advance, and the model considers not only the location 
problem but also the quantity of interception raw materials and products flows. This model will then be applied in a case study in the further study.

\section{REFERENCES}

Berman, O., Larson, R. C., \& Fouska, N. (1992). Optimal location of discretionary service. Transportation Science, 26(3), 201-211. doi: 10.1287/trsc.26.3.201

Fang, Y., \& Ng, S. T. (2011). Applying activity - based costing approach for construction logistics cost analysis. Construction Innovation, 11(3), 259-281. doi: doi:10.1108/14714171111149007

Gürel, S. (2011). A conic quadratic formulation for a class of convex congestion functions in network flow problems. European Journal of Operational Research, 211(2), 252-262. doi: http://dx.doi.org/10.1016/j.ejor.2010.12.018

Hodgson, M. J. (1990). A flow-capturing location-allocation model. Geographical Analysis, 22(3), 270-279.

Hu, D., Yang, C., \& Yang, J. (2009). Budget constrained flow interception location model for congested systems. Journal of Systems Engineering and Electronics, 20(6), 1255-1262.

Jaillon, L., \& Poon, C. S. (2008). Sustainable construction aspects of using prefabrication in dense urban environment: a Hong Kong case study. Construction Management and Economics, 26(9), 953-966. doi: 10.1080/01446190802259043

khaled, a. m., \& Kim, Y. J. (2012). GIS Exploitation for New Facility Location Decisions- A Logistics Perspective. Journal of Intl Logistics and Trade, 10(1), 43-62.

li, C., Zhu, L., Zhang, N., \& Hu, Z. (2011). Shenyang Modern Architecture Industrial Park Planning Base the Circular Economy Theory. Planners, 27(C00), 234-237.

MOHURD. (2015). The awarding ceremony of National Housing Industrialization pilot cities and bases was held in Beijing. from http://www.chinahouse.gov.cn/zzbp5/z1720.htm

Ning, W., \& Yingbo, J. (2010, 26-28 Nov. 2010). Analysis on Key Factors to Affect Construction Industrialization Development in China. Paper presented at the 2010 3rd International Conference on Information Management, Innovation Management and Industrial Engineering.

Rahman, M. M. (2014). Barriers of Implementing Modern Methods of Construction. Journal of Management in Engineering, 30(1), 69-77.

Wang, X. (2008). Locating competitive service facilities in uncertain market using the flow intercepting spatial interaction model. Paper presented at the 2008 International Conference on Wireless Communications, Networking and Mobile Computing, WiCOM 2008, October 12, 2008 - October 14, 2008, Dalian, China.

Wu, S., Yang, Z., \& Dong, X. (2014). Optimization Method for Location and Distribution of Manufacturing Industry Based on the Flow-Capturing Location Model. Economic Geography, 34(11), 95-100.

Wu, S., Yang, Z., \& Dong, X. (2015). Optimization methods for location and distribution of manufacturing industry based on the bi-level programming model. Systems EngineeringTheory \& Practice, 35(11), 2840-2848.

Zeng, W. P., Castillo, I., \& Hodgson, M. J. (2010). A Generalized Model for Locating Facilities on a Network with Flow-Based Demand. Networks \& Spatial Economics, 10(4), 579-611. doi: 10.1007/s11067-008-9073-8 